\title{
BMJ Open Awareness and attitudes towards advance care planning in primary care: role of demographic, socioeconomic and religiosity factors in a cross-sectional Lebanese study
}

Georges Assaf, ${ }^{1,2}$ Sarah Jawhar, ${ }^{1}$ Kamal Wahab, ${ }^{1}$ Rita El Hachem, ${ }^{3}$ Tanjeev Kaur, ${ }^{2}$ Maria Tanielian, ${ }^{1}$ Lea Feghali, ${ }^{1}$ Adina Zeki Al Hazzouri, ${ }^{4}$ Martine Elbejjani (D ${ }^{3}$

To cite: Assaf G, Jawhar S, Wahab K, et al. Awareness and attitudes towards advance care planning in primary care: role of demographic, socioeconomic and religiosity factors in a cross-sectional Lebanese study. BMJ Open 2021;11:e052170. doi:10.1136/ bmjopen-2021-052170

- Prepublication history and additional supplemental material for this paper are available online. To view these files, please visit the journal online (http://dx.doi.org/10.1136/ bmjopen-2021-052170).

Received 07 April 2021 Accepted 13 October 2021

Check for updates

(c) Author(s) (or their employer(s)) 2021. Re-use permitted under CC BY-NC. No commercial re-use. See rights and permissions. Published by BMJ.

For numbered affiliations see end of article.

Correspondence to Dr Martine Elbejjani; me158@aub.edu.lb

\section{ABSTRACT}

Objective To assess awareness and attitudes towards engaging in advance care planning (ACP) and their relationship with demographic, socioeconomic and religiosity factors among Lebanese middle-aged to olderaged adults in primary care.

Design A cross-sectional survey study.

Setting Tertiary referral hospital in Beirut, Lebanon. Participants A total of 215 middle-aged to older-aged adults.

Results Out of 215 participants, $18.6 \%$ of participants knew about ACP; 94\% favoured truth-telling; $87.4 \%$ favoured healthcare autonomy; $77.2 \%$ favoured documenting their own health values and preferences; and $29.3 \%$ were willing to undergo life-prolonging interventions. Among participants who were aware of ACP, $67.5 \%$ preferred ACP documentation and $85 \%$ had negative attitudes towards life-sustaining interventions. Women were more aware about ACP than men. Those who were willing to undergo life-prolonging interventions were found to be men and had higher religiosity scores. Conclusion Large deficit in ACP awareness was evident despite the high preference for healthcare autonomy. Medical and public health efforts should strive to enhance patients' ACP awareness and engagement in ACP while considering factors relevant to gender, culture and religiosity.

\section{INTRODUCTION}

Advance care planning (ACP) is a process aiming to support adults at any age or stage of health in receiving medical care that is aligned with their personal values, goals and preferences. ${ }^{12}$ One important component of ACP is to help people in their healthcare decisions and wishes for their end of life (EOL) care in the event their decision-making capacity becomes impaired. ${ }^{3-5}$ It involves communication between patients, healthcare providers, family members and care partners to plan ahead for medical care options and preferences at EOL. ${ }^{3}$ This process may lead

\section{STRENGTHS AND LIMITATIONS OF THIS STUDY}

$\Rightarrow$ This is the first study to provide data that addresses gaps in the literature regarding advance care planning (ACP) among middle-aged to older-aged adults visiting a primary care setting in the Middle Eastern region.

$\Rightarrow$ The data were based on a small convenience sample of participants who have access to a large private academic medical centre in the capital which limits the generalisability of our findings.

$\Rightarrow$ The instrument used to assess the awareness and attitude towards ACP had not yet been validated in the Lebanese context and may have failed to accurately measure several constructs such as healthcare autonomy since the perception of patient autonomy and decision making may differ among cultures.

to ongoing discussions and/or documentation of an advance directive, which reflects patients' wishes for future medical decisions if they become unable to make such decisions. ${ }^{56}$

As we encounter an ageing population, an increase in chronic medical conditions and terminal illnesses, engagement in ACP has important advantages for ensuring patientcentred care and improved EOL care quality. ACP enables improved EOL communication, documentation of care preferences, selection of environment during death and discussion of healthcare costs and expenditures and resource/savings allocation. ${ }^{78} \mathrm{ACP}$ in EOL care has been found to reduce unnecessary medical interventions, prevent hospital admissions and increase the use of hospice and palliative care. ${ }^{79}$ It is also associated with reduced suffering and greater satisfaction for both patients and family members who also 
benefit from experiencing lower levels of stress, anxiety and depression. ${ }^{710}$

Despite the increasingly recognised importance of ACP, previous studies have shown that both patients and healthcare professionals face barriers in engaging in ACP which may contribute to the low completion rate of advance directives in general and specific patient populations like those in primary care. ${ }^{11-13}$ Primary care physicians are well placed to facilitate and initiate ACP discussion given their role in care coordination of chronic and terminal illnesses care and their close and longer-term relationship with their patients; however, the number of patients who are actually engaged in ACP remains low. ${ }^{14}$ One key barrier among patients is the lack of knowledge and negative perceptions regarding ACP. ${ }^{15} 16$ Therefore, determining patients' knowledge and attitudes towards ACP is instrumental for the development of effective strategies to facilitate EOL care discussions and decision making and promote public awareness regarding ACP especially in primary care settings. ${ }^{11} 1718$ Cultural factors and values also influence patients' attitude towards discussing serious illness and willingness to participate in ACP. ${ }^{19}{ }^{20}$ Indeed, prior research has shown that engagement in ACP and completing advance directives, as well as their barriers and facilitators, vary across populations and ethnic groups. ${ }^{21-23}$ Notably, several studies also show that religiosity plays an important role in ACP preferences, with studies reporting that individuals with greater religiosity are less likely to engage in ACP and complete advance directives and less willing to forego life-sustaining treatments at EOL. ${ }^{24-26}$ However, to date, data on ACP knowledge and preferences are scarce in Middle-Eastern countries and the influence of religious factors-which play a central role in Middle-Eastern communities-on these preferences are largely understudied. ${ }^{27}$

In Lebanon, the population is experiencing a fast rate of ageing where adults over the age of 65 are expected to constitute $31.2 \%$ of the population by the year $2050 .^{28}$ Similarly, there is an increase in the prevalence of chronic and terminal illnesses which emphasises the need for promoting ACP across the continuum of care in the country. ${ }^{29}{ }^{30}$ Lebanon is also a religiously diverse country with both large and diverse Muslim and Christian communities, and where religion plays an important role in people's lives. ${ }^{27}$ 31-35 In addition, the country has frequently endured economic instability imposing important socioeconomic constraints on peoples' livelihood including their healthcare. To date, there is a paucity of data on the awareness about ACP and preferences for EOL care in the Lebanese population.

This study aimed to assess the awareness and attitudes towards ACP among Lebanese adult patients visiting a primary care centre and to evaluate whether awareness and attitudes are related to demographic, socioeconomic and religiosity factors. To provide further insight into ACP preferences in this population, we also assessed whether dementia knowledge and attitudes towards people with dementia are associated with ACP awareness and attitudes, evaluating how people's knowledge and perceptions of this severely incapacitating illness was linked to ACP.

\section{METHODS}

\section{Study design, setting and participant recruitment}

This is a cross-sectional study in which participants were recruited as part of a larger survey conducted in 2019 that included two components: (1) investigating the knowledge and attitudes of patients towards Alzheimer's Disease and Related Dementias (ADRD) using the Alzheimer's Disease Knowledge Scale (ADKS) and (2) investigating knowledge and attitudes towards ACP. Participants aged 45 years and older with no severe uncorrected hearing impairment (no severe hearing impairment or participants with impairment but having or using hearing aids) or visual impairment or aphasia were approached in the primary care centre's triage area at the American University of Beirut Medical Center, Beirut, Lebanon. Participants' recruitment occurred during clinic hours on both weekdays and weekends. Participants completed a standardised interviewer-based questionnaire that was administered by trained data collectors. All participants were assured that their participation would be voluntary and anonymous and would not affect the health services provided to them. All participants provided written informed consent. In total, 215 participants completed the study questionnaire.

\section{Patient and public involvement statement}

Participants were not involved in the design, or conduct, or reporting, or dissemination plans of this research.

\section{Measurements}

The study questionnaire included two sections. The first section included sociodemographic characteristics of the participant; age (years), gender (man/woman), marital status (married/ not married), educational attainment (lower than university degree/at least a university degree), current employment (yes/no), monthly household income (lower: $\leq 2$ million Lebanese pounds (LBP)/ high: LBP 2 million or $>$ US\$1300) and current smoking status (yes/no). The second section was based on a previously published 5-item questionnaire that evaluated participants' awareness and attitudes towards ACP: 'In case you were diagnosed with cancer or Alzheimer's disease, do you want to hear the real news regarding your own condition from the physician (Q1)?'; 'Would you prefer to make your own healthcare decisions (Q2)?'; 'Have you ever heard of an Advance Care Planning (Q3)?'; 'Do you want to document your own values and preferences related to your health and treatment options so that it will be respected in case you become seriously ill (Q4)?'; 'Are you willing to endure specific life-prolonging interventions (such as chronic ventilator and feeding tube) to avoid death when faced with irreversible conditions (Q5)?? ${ }^{36}$ The first two questions aimed to assess 
participants' attitudes towards truth-telling (disclosure of diagnosis and prognosis about patients' medical condition from physician) and healthcare autonomy. Question 3 evaluated participant's awareness about ACP, question 4 related to engagement in and preferences towards ACP, and question 5 assessed participants' attitudes towards end-of-life care. ${ }^{36}$ Participants were first asked questions 1, 2 and 3. These questions required a response of 'yes' or 'no'. For those who were not aware of the concept of ACP (ie, answering 'No' to question 3), the interviewers explained the concept before moving on to asking questions 4 and 5. The English version of the questionnaire was translated into Arabic by a professional translator with input and feedback from the research team; the ACP questionnaire is presented in online supplemental table 1 .

Given the importance of religiosity in the Lebanese culture and in shaping one's perception of the causes and consequences of disease in general and of ACP and EOL care in particular, ${ }^{2425}{ }^{27}$ we assessed religiosity indicators using the Duke Religion Index (DUREL), translated into Arabic. The DUREL measures three dimensions of religiosity: The organisational item measures frequency of attendance at religious services (1: never, 2: once a year or less, 3: a few times a year, 4: a few times a month, 5 : once a week, 6: >once a week); the non-organisational item assesses the frequency of private religious activities such as prayer or meditation (1: rarely or never, 2 : a few times a month, 3: once a week, 4: 2 or more times a week, 5: daily, 6: >once a day). The organisational and non-organisational religious activities items each generate a score from 1 to 6 with a higher number indicating a higher frequency of religious activities. Detailed frequency distributions of the DUREL religious activity items in the sample are presented in online supplemental table 1. Intrinsic religiosity is measured with three items that ask about the agreement with statements about 'experiencing the presence of the divine', 'allowing religious beliefs to lie behind one's whole approach to life', and 'trying hard to carry religion over into all other areas of life' (scored from 1: definitely not true to 5: definitely true). The intrinsic religiosity dimension is a sum of the three intrinsic religiosity items with higher scores indicating higher intrinsic religiosity. ${ }^{37}$

In an additional analysis, we assessed whether ADRD knowledge, attitudes towards people with dementia, and having a family or a friend with dementia and/or memory problems were related to awareness and attitudes towards ACP. The motivation for this analysis was to provide more insight into factors related to ACP, given the paucity of data in the Lebanese population. As detailed elsewhere, ${ }^{38}$ ADRD knowledge was assessed using the ADKS which consists of 30 true/false items. ${ }^{38}{ }^{39}$ For each participant, the total knowledge score was computed by summing the 30 items of the ADKS with each correct answer being one additional point, thus higher scores indicate higher knowledge. Attitudes towards people with dementia were evaluated using an eight-item questionnaire, ${ }^{40}$ with a positive attitude answer for each item scored as one point; these items were summed to obtain a total score ranging from 0 to 8 , with higher values indicating more positive attitudes towards people with dementia. ${ }^{39} 40$ Through medical and language translation and adaptation, the research team generated Arabic versions of the ADKS and the dementia-related attitudes questionnaire. ${ }^{38}$ Participants also reported whether they had a family member or a friend diagnosed with dementia and whether they had a family member or friend who had severe memory problems that were not diagnosed (yes/no); including the latter category aimed to capture potential experiences with dementia in participant's social network that did not receive proper diagnosis. ${ }^{38}$

\section{STATISTICAL ANALYSIS}

ACP items were described individually in percentages. We assessed whether awareness about ACP (item 3) was associated with the perception items (items $1-2,4-5)$ using $\chi^{2}$ tests. Using $\chi^{2}$ tests for categorical variables and independent $t$-test for continuous variables, we evaluated whether demographic, socioeconomic and religiosity factors were different across each of the five ACP items. Using Fisher's exact test for comparisons of categorical variables produced similar conclusions as the $\chi^{2}$ tests. All analyses were performed with the SAS statistical package (V.9.4; SAS Institute) and SPSS Statistics V.24 (SPSS). All results were considered statistically significant at $\mathrm{p}<0.05$.

\section{RESULTS}

\section{Sociodemographic characteristics}

Characteristics of the 215 participants are presented in table 1 . The mean age was 58.6 years $( \pm 9.6), 58.1 \%$ were

Table 1 Characteristics of participants $(n=215)$

\begin{tabular}{lll}
\hline Characteristics & $\begin{array}{l}\text { Mean (SD) } \\
\text { or } \mathbf{n}(\%)\end{array}$ & $\begin{array}{l}\text { Missing } \\
\text { data } \mathbf{~ ( \% ) ~}\end{array}$ \\
\hline Age (years) (range: 40-91) & $58.65(9.64)$ & $6(2.7)$ \\
\hline Gender, women & $125(58.1)$ & 0 \\
\hline Current smoker, daily & $71(33)$ & $4(1.8)$ \\
\hline Marital status, married & $175(82.5)$ & $3(1.3)$ \\
\hline $\begin{array}{l}\text { Educational attainment, } \\
\geq \text { university level }\end{array}$ & $92(42.8)$ & $2(0.9)$ \\
\hline Employment, yes & $127(59.1)$ & $2(0.9)$ \\
\hline $\begin{array}{l}\text { Income, high (>LBP2 million) } \\
\text { DUREL subscales }\end{array}$ & $105(48.8)$ & $26(12)$ \\
\hline $\begin{array}{l}\text { Organisational religious } \\
\text { activity }\end{array}$ & $2.97(1.79)$ & 0 \\
\hline $\begin{array}{l}\text { Non-organisational religious } \\
\text { activity }\end{array}$ & $4.36(1.84)$ & 0 \\
\hline $\begin{array}{l}\text { Intrinsic religiosity } \\
\text { Intion }\end{array}$ & $12.27(3.22)$ & $1(0.4)$ \\
\hline
\end{tabular}

DUREL, Duke University religion index; LBP, lebanese pounds. 


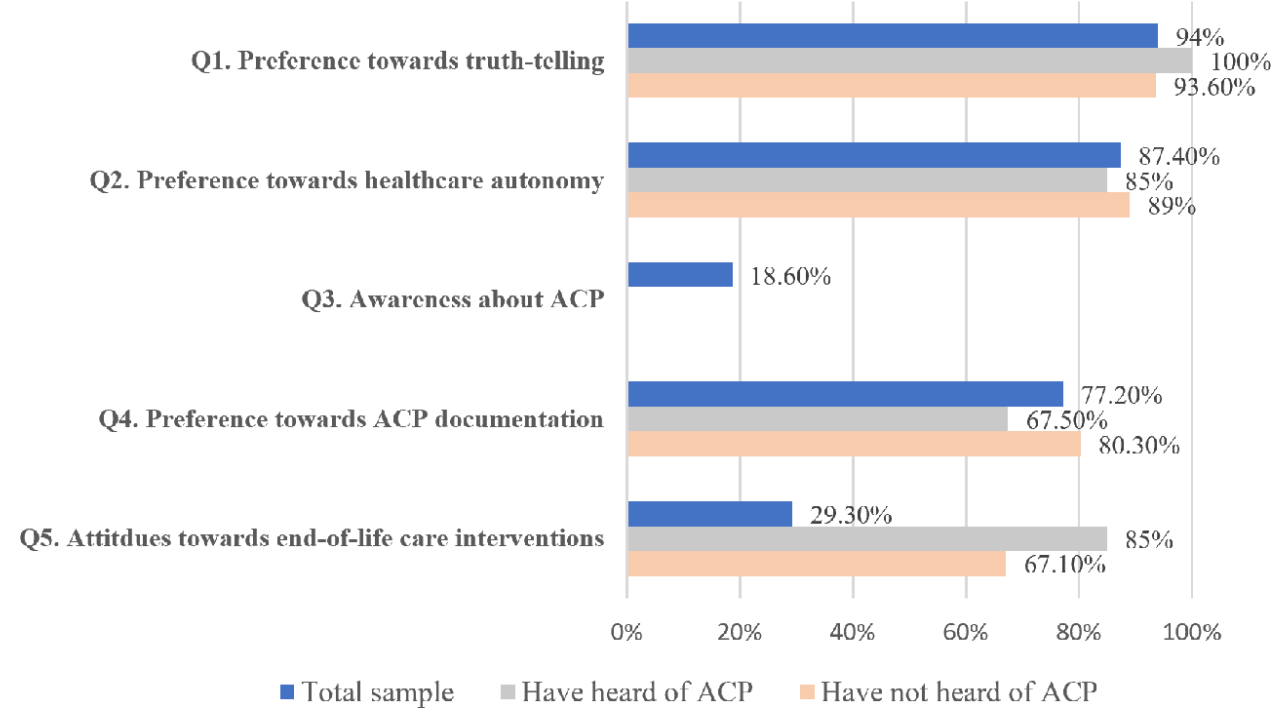

Figure 1 Awareness and attitudes towards advance care planning (ACP) among Lebanese primary care patients, in the total sample and among those who have heard and those who have no theard about $A C P, n=215$. Bar charts present the proportions of yes answers to each item. P values of $\chi 2$ tests comparing proportions of ACP preference and perception items Q1, Q2, Q4, Q5 between participants who have heard of ACP and those who have not, respectively: $0.13,0.59,0.09,0.03$.

female, $82.5 \%$ were married, $42.8 \%$ had higher educational attainment, $59.1 \%$ were employed and $48.8 \%$ had a monthly household income of more than LBP2 million $(\sim \mathrm{US} \$ 1300)$.

\section{Religiosity-related variables}

The average DUREL scores were $3.0 \quad(\mathrm{SD}=1.8), 4.4$ $(\mathrm{SD}=1.8)$ and $12.3(\mathrm{SD}=3.2)$ for the organisational religious activities, non-organisational religious activities and intrinsic religiosity, respectively.

\section{Awareness and attitudes of participants towards ACP}

In this sample, $18.6 \%$ of participants had heard of ACP. Regarding ACP attitudes and preferences, $94 \%$ of participants reported that they would like to hear the real news regarding their own condition from the physician and $87.4 \%$ reported that they prefer to make their own healthcare decisions. The majority $(77.2 \%)$ reported that they would want to document their own values and preferences regarding their health and treatment decisions to be respected in case they become seriously ill and 29.3\% reported being willing to endure specific life-prolonging interventions (such as chronic ventilator and feeding tube) to avoid death when faced with irreversible conditions (figure 1 and online supplemental table 1).

Figure 1 also presents the distribution of ACP attitudes and preferences among participants who have heard of ACP and those who have not. Participants who have heard of ACP had a higher preference for not going through life-prolonging interventions when faced with irreversible conditions $(85 \%$ vs $67.1 \%$ among those not heard of ACP; $p$ value of $\chi^{2}$ test comparing these two groups $=0.03$ ). These two groups were similar with regards to preferences for knowing their medical diagnosis $(p=0.13)$, making their own health decisions $(\mathrm{p}=0.59)$ and for documenting healthcare preferences $(\mathrm{p}=0.09)$.

\section{Sociodemographic and socioeconomic factors and ACP}

There were statistically significant gender differences in awareness about ACP as well as attitude towards EOL care interventions: Women had higher ACP awareness $(24.2 \%)$ than men $(11.2 \%)$ and were less $(24.2 \%)$ willing to endure specific life-prolonging interventions (such as chronic ventilator and feeding tube) than men $(37.1 \%$; table 2).

There were no differences in ACP knowledge and attitudes across age and marital status, education, income and employment categories (table 2).

\section{Religiosity and ACP}

There were differences in religiosity scores across ACP preferences. Participants who were willing to endure life-prolonging interventions had higher religiosity scores than those who were not (specifically they had higher means scores of non-organisational religious activities $=4.9(1.5)$ vs $4.2(1.9)$, t-test $\mathrm{p}=0.01$ and intrinsic religiosity $=12.9$ (2.6) vs 12.1 (3.4); $\mathrm{p}=0.09)$; participants who reported no preference for documenting their healthcare values and options also tended to have higher religiosity scores (mean scores for intrinsic religiosity=13.1 (2.7) vs 12.1 (3.3); $\mathrm{p}=0.1$ and organisational religious activities $(3.3$ (1.6) vs $2.9(1.8) ; \mathrm{p}=0.1$ (table 2)).

Online supplemental table 2 presents the detailed distribution of categories of frequency of engagement in nonorganisational and organisational religious activity and the ACP items and shows similar patterns of results, with a higher frequency of non-organisational religious activity among participants willing to endure life-prolonging interventions and among participants reporting no preference for healthcare autonomy; participants reporting no preference for ACP documentation also tended to 


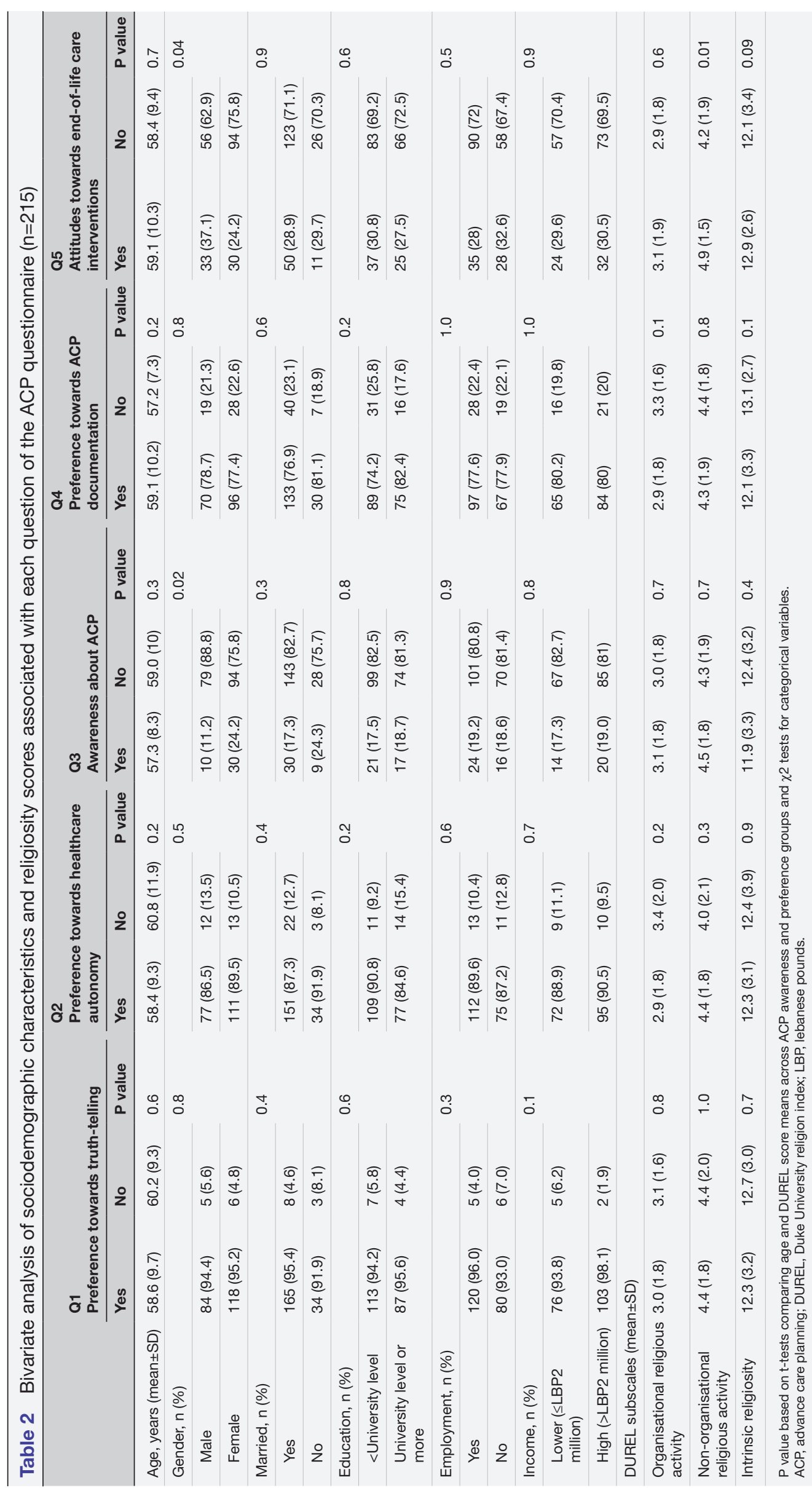


have higher frequency of engagement in organisational religious activity.

\section{Dementia-related knowledge and attitudes and ACP}

In additional analysis, we assessed whether ADRD knowledge and attitude towards people with dementia were related to ACP awareness and attitudes. Overall, the mean dementia knowledge score was $18.7( \pm 3.1)$ and the mean attitude score towards dementia was $5.8( \pm 1.6)$ indicating low to moderate knowledge about ADRD and positive attitudes towards people with dementia. More than half $(56.4 \%)$ of participants had a family member or friends with dementia or memory problems: $27 \%$ had a family member with dementia and $15 \%$ had a friend with dementia, while $29 \%$ of participants had a family member and $17 \%$ had a friend with severe memory problems (online supplemental table 3).

Participants preferring to document their healthcare values and preferences had significantly higher ADRD knowledge (19.1 (3.0) vs 17.2 (3.2); $\mathrm{p} \leq 0.0001$; table 3). There was a significant difference in willingness to endure life-prolonging interventions between those who had a family member/friend with dementia/memory problems and those who did not ( $(22.9 \%$ vs. $37.4 \%$ willing, respectively; $\mathrm{p}=0.03$; table 3 ). Attitudes towards people with dementia were not different across the ACP items, except for a trend for participants who have heard of ACP to have more positive attitudes towards people with dementia (attitude mean scores among those aware and not aware of ACP, respectively: 6.2 (1.2) vs 5.7 (1.6); $\mathrm{p}=0.09$ ).

\section{DISCUSSION}

To the best of our knowledge, this is the first study to investigate the preferences and attitudes towards ACP among middle-aged to older-aged participants attending primary care in Lebanon. Our findings showed a high preference for healthcare autonomy in this sample despite a significantly low level of awareness regarding ACP, motivating future efforts to raise awareness about ACP and to integrate it into patients' care plans. Our findings revealed that women were more aware of ACP and less willing to undergo life-prolonging interventions when faced with irreversible conditions compared with men. Religiosity was also related to attitudes towards ACP, with participants with higher religiosity scores showing higher preference for life-prolonging interventions and lower preference towards documenting their healthcare values and decisions. Participants with higher knowledge, positive attitudes and social experience related to ADRD showed a higher preference for ACP documentation and were less willingness to endure life-prolonging interventions in EOL.

The Lebanese society is known to have a family-oriented culture where decisions, including healthcare, are made in conjunction with other family members which potentially lessens the value of autonomy. ${ }^{41}{ }^{42}$ However, most of the participants in our study favoured truth-telling,

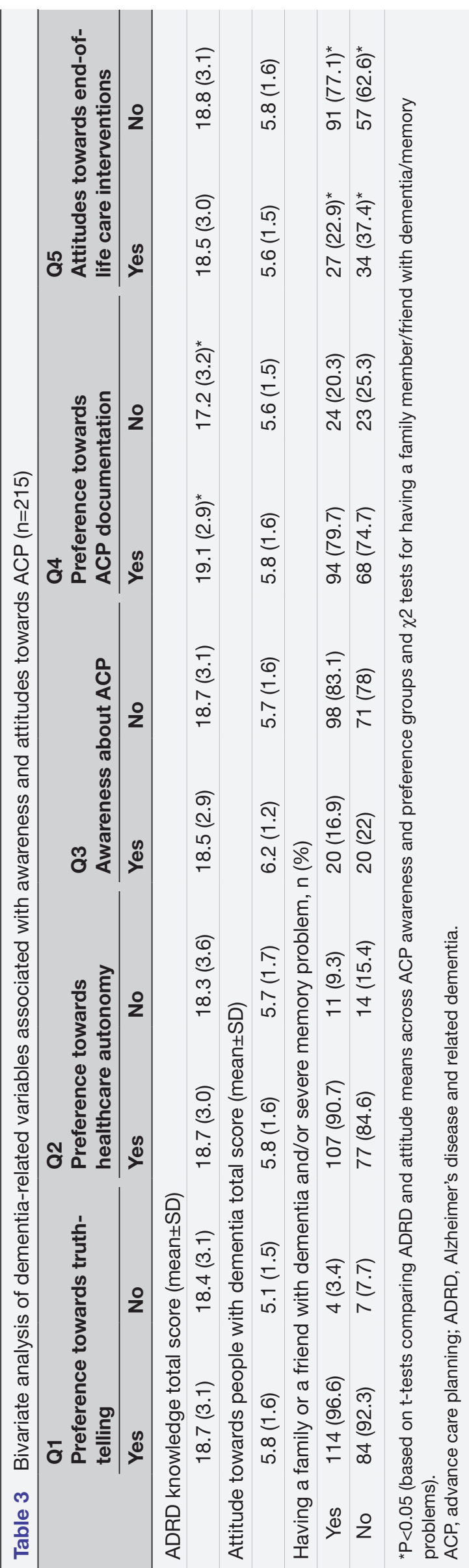


healthcare autonomy and making their own healthcare decisions, suggesting an attitude that favours active participation in care management; this is in line with findings from another study that showed that Lebanese patients prefer taking part in the management of their own treatments. ${ }^{43}$ These findings that Lebanese adults show preference towards a more engaged and independent decision-making process can be explained by the increased accessibility and advancement in education and technology. ${ }^{44}$

Our study revealed a large deficit in knowledge about ACP, which can be attributed to several reasons. While ACP is widely practised in Western societies, the concept is still relatively new in the Middle Eastern region and major differences exist between this region and Western countries with regards to advance directives, decision making, life support, religious and cultural perception of palliative care, and ACP. ${ }^{21445}$ For example, the Lebanese code of medical ethics still lacks clarity on the concept of advance directives and life-sustaining medical treatment. Consequently, ACP discussions might not be commonly practised by physicians due to the absence of clear guidelines on how to implement a patient's will in the event of becoming ill or hospitalised, which in turn explains the low level of awareness about ACP among patients. Yet, despite not formally knowing about ACP, our sample showed elevated preferences towards documenting their healthcare values and options and to make their own healthcare decisions. Previous studies also revealed patients' preferences towards discussing advance directives early in the patient-physician relationship. ${ }^{46}$ In countries like the USA, UK and France, ACP discussions are always raised in outpatient settings and tailored based on patient's preferences whereas, in Lebanon, palliative care for instance is only initiated for a patient if all other means of curative treatments have failed regardless of whether patients' wishes have been documented before illness. ${ }^{45}$

While the majority of the participants did not opt for life-prolonging interventions, those who have heard of ACP were less willing to opt for such interventions than those who have not heard of ACP. This finding is consistent with previous studies that showed that ACP influences the prevention or reduction of the use of life-sustaining treatments. ${ }^{89}$ An earlier study conducted in a Lebanese sample reported that patients and their families tend to opt for treatments that prolong life, even if that means suffering from severe physical and psychological pain. ${ }^{44}$ Preferences for life-sustaining interventions also differ based on social influences, cultural and religious beliefs. In our sample, women were less likely to be willing to endure life-prolonging treatment than men; this finding was reported in other populations and is thought to be probably due to women's perceptions of these measures as a burden on their future caregivers (most of the time it is their adult children) and wanting to relieve them from this responsibility. ${ }^{47-49}$ Meanwhile, those who reported willingness to endure life-prolonging interventions had higher religiosity scores, in line with previous studies on religion, EOL decision making and ACP where patients with higher religious beliefs were more likely to choose life-long measures. ${ }^{50-52}$ This relationship was observed with higher intrinsic religiosity and engagement in nonorganisational religious activity; lower preference for ACP documentation was also related to higher intrinsic religiosity. This is in line with other reports finding that specific aspects of religiosity relate differently to ACP; for example, higher religious belief was inversely associated with advance directive completion, whereas higher attendance of religious services was positively associated with directive completion. ${ }^{24}$ Together, these findings could reflect the importance of religious beliefs as well as the notion that individual religious activity may be a way of coping with illness and stress and a response to potential adverse health consequences ${ }^{37}$; similarly, they could be reflecting a possibly better health status and potential positive social and community benefits of attending religious services. Notably, our sample had elevated religiosity scores, particularly for non-organisational religious activities and intrinsic religiosity, warranting further considerations and investigations of the role of religious and personal beliefs in ACP discussions in this population.

As for dementia-knowledge-related factors, participants who had heard of ACP and had favoured healthcare autonomy had more positive attitudes towards people with dementia and higher dementia knowledge scores, respectively. This could be explained by a general and/ or common higher knowledge and more positive experiences and perceptions of health-related issues and chronic illnesses. Also, participants who had a family member or a friend with dementia/memory problems were found unfavourable of life-prolonging interventions. It is possible that personal experience with the burdensome complications of serious illness may lead individuals to oppose life-sustaining treatment in a similar situation. ${ }^{49}$ Future investigations would benefit from further understanding the interplay between different religiosity aspects, health status and experiences, and awareness and attitudes towards ACP.

This study has several limitations such as the small convenience sample and potential recruitment of participants who may have a higher socioeconomic status and have access to a large private academic medical centre in the capital which limits the generalisability of our findings. Multimorbidity was not incorporated into the study, which may also be an important determinant in ACP and its perceived relevance to individuals. The instrument used to assess the awareness and attitude towards ACP had not yet been validated in the Lebanese context and may have failed to accurately measure several constructs such as healthcare autonomy since the perception of patient autonomy and decision making may differ among cultures. 


\section{CONCLUSION}

Our findings revealed a large deficit in awareness about ACP among Lebanese middle-aged to older-aged adults in primary care. Yet, the majority of participants seemed to favour healthcare autonomy and documentation of ACP preferences along with having negative attitudes towards life-prolonging treatments. Therefore, there's a need for improved awareness and applicability of ACP through establishing medical guidelines and effective public awareness campaigns while considering gender and religiosity factors and personal experiences with illness.

\section{Author affiliations}

${ }^{1}$ Department of Family Medicine, American University of Beirut Medical Center, Beirut, Lebanon

${ }^{2}$ Department of Academic Internal Medicine and Geriatrics, University of Illinois at Chicago, Chicago, Illinois, USA

${ }^{3}$ Clinical Research Institute, Department of Internal Medicine, American University of Beirut, Beirut, Lebanon

${ }^{4}$ Epidemiology, Columbia University Mailman School of Public Health, New York, New York, USA

\section{Twitter Martine Elbejjani @martinebejjani}

Contributors This study was designed, directed and coordinated by GA and ME (study guarantor). KW, MT and LF contributed to data collection. ME and REH were responsible for conducting data analysis. SJ, MT, GA, ME, and LF drafted the manuscript. GA, SJ, KW, REH, TK, MT, LF and AZAH contributed to the interpretation of the data, revising the manuscript critically for important intellectual content and to shaping the research and final manuscript, and approved the final manuscript.

Funding The authors have not declared a specific grant for this research from any funding agency in the public, commercial or not-for-profit sectors.

Competing interests None declared.

Patient consent for publication Consent obtained directly from patient(s)

Ethics approval The study protocol was approved by the American University of Beirut Institutional Review Board (SBS-2017-0598).

Provenance and peer review Not commissioned; externally peer reviewed.

Data availability statement Data are available on reasonable request. The data include deidentified participant data that is available upon request from the corresponding author Dr. Martine Elbejjani (ORCID: 0000-0002-5035-0370).

Supplemental material This content has been supplied by the author(s). It has not been vetted by BMJ Publishing Group Limited (BMJ) and may not have been peer-reviewed. Any opinions or recommendations discussed are solely those of the author(s) and are not endorsed by BMJ. BMJ disclaims all liability and responsibility arising from any reliance placed on the content. Where the content includes any translated material, BMJ does not warrant the accuracy and reliability of the translations (including but not limited to local regulations, clinical guidelines, terminology, drug names and drug dosages), and is not responsible for any error and/or omissions arising from translation and adaptation or otherwise.

Open access This is an open access article distributed in accordance with the Creative Commons Attribution Non Commercial (CC BY-NC 4.0) license, which permits others to distribute, remix, adapt, build upon this work non-commercially, and license their derivative works on different terms, provided the original work is properly cited, appropriate credit is given, any changes made indicated, and the use is non-commercial. See: http://creativecommons.org/licenses/by-nc/4.0/.

\section{ORCID iD}

Martine Elbejjani http://orcid.org/0000-0002-5035-0370

\section{REFERENCES}

1 Sudore RL, Lum HD, You JJ, et al. Defining advance care planning for adults: a consensus definition from a multidisciplinary Delphi panel. J Pain Symptom Manage 2017;53:821-32.
2 Peicius E, Blazeviciene A, Kaminskas R. Are advance directives helpful for good end of life decision making: a cross sectional survey of health professionals. BMC Med Ethics 2017;18:1-7.

3 Committee on Approaching Death: Addressing Key End of Life Issues; Institute of Medicine. Dying in America: improving quality and honoring individual preferences near the end of life. Washington (DC): National Academies Press (US), 2015.

4 Levi BH, Green MJ. Too soon to give up: re-examining the value of advance directives. The American Journal of Bioethics 2010;10:3-22.

5 National Institute on Aging. Advance care planning: tips from the National Institute on Aging [Internet]. National Institute of Health, 2018. Available: https://order.nia.nih.gov/sites/default/files/2018-03/ advance-planning-tip-sheet.pdf

6 Howard M, Bernard C, Tan A. Advance care planning: let's start sooner. Canadian Family Physician 2015;61:663-5.

7 Wright AA, Zhang B, Ray A, et al. Associations between end-of-life discussions, patient mental health, medical care near death, and caregiver bereavement adjustment. JAMA 2008;300:1665-73.

8 Jimenez G, Tan WS, Virk AK, et al. Overview of systematic reviews of advance care planning: summary of evidence and global lessons. $J$ Pain Symptom Manage 2018;56:436-59.

9 Brinkman-Stoppelenburg A, Rietjens JAC, van der Heide A. The effects of advance care planning on end-of-life care: a systematic review. Palliat Med 2014;28:1000-25.

10 Detering KM, Hancock AD, Reade MC, et al. The impact of advance care planning on end of life care in elderly patients: randomised controlled trial. BMJ 2010;340:c1345.

11 Schickedanz AD, Schillinger D, Landefeld CS, et al. A clinical framework for improving the advance care planning process: start with patients' self-identified barriers. J Am Geriatr Soc 2009;57:31-9.

12 Ramsaroop SD, Reid MC, Adelman RD. Completing an advance directive in the primary care setting: what do we need for success? J Am Geriatr Soc 2007;55:277-83.

13 Tung EE, North F. Advance care planning in the primary care setting: a comparison of attending staff and resident barriers. Am J Hosp Palliat Care 2009;26:456-63.

14 De Vleminck A, Houttekier D, Pardon K, et al. Barriers and facilitators for general practitioners to engage in advance care planning: a systematic review. Scand J Prim Health Care 2013;31:215-26.

15 Bernard C, Tan A, Slaven M, et al. Exploring patient-reported barriers to advance care planning in family practice. BMC Fam Pract 2020;21:1-9.

16 Blackwood DH, Walker D, Mythen MG, et al. Barriers to advance care planning with patients as perceived by nurses and other healthcare professionals: a systematic review. $J$ Clin Nurs 2019;28:4276-97.

17 Kleiner AC, Santos-Eggimann B, Fustinoni S. Advance care planning dispositions: the relationship between knowledge and perception. BMC Geriatr 2019;19:1-11.

18 Kermel-Schiffman I, Werner P. Knowledge regarding advance care planning: a systematic review. Arch Gerontol Geriatr 2017;73:133-42.

19 Searight HR, Gafford J. Cultural diversity at the end of life: issues and guidelines for family physicians. Am Fam Physician 2005;71:515-22.

20 Perkins HS, Geppert CMA, Gonzales A, et al. Cross-Cultural similarities and differences in attitudes about advance care planning. $J$ Gen Intern Med 2002;17:48-57.

21 Johnstone M-J, Kanitsaki O. Cultural racism, language prejudice and discrimination in hospital contexts: an Australian study. Diversity in Health and Social Care 2008;5:19-30.

22 Hong M, Yi E-H, Johnson KJ, et al. Facilitators and barriers for advance care planning among ethnic and racial minorities in the U.S. a systematic review of the current literature. J Immigr Minor Health 2018;20:1277-87.

23 Carr D. Racial and ethnic differences in advance care planning: identifying subgroup patterns and obstacles. Journal of Aging and Health 2012;24:923-47.

24 Hoe DF, Enguidanos S, Me SH. So help me, God: religiosity and endof-life choices in a nationally representative sample. J Palliat Med 2020;23:563-7.

25 Choudry M, Latif A, Warburton KG. An overview of the spiritual importances of end-of-life care among the five major faiths of the United Kingdom. Clin Med 2018;18:23-31.

26 Garrido MM, Idler EL, Leventhal $\mathrm{H}$, et al. Pathways from religion to advance care planning: beliefs about control over length of life and end-of-life values. Gerontologist 2013;53:801-16.

27 Gebara J, Tashjian H. End-Of-Life practices at a Lebanese Hospital: courage or knowledge? J Transcult Nurs 2006;17:381-8.

28 United Nations Department of Economic and Social Affairs. World population ageing 2017 - highlights (ST/ESA/SER.A/397), 2017. Available: https://www.un.org/en/development/desa/population/ publications/pdf/ageing/WPA2017_Highlights.pdf 
29 Silbermann M, Fink RM, Min S-J, et al. Evaluating palliative care needs in middle Eastern countries. J Palliat Med 2015;18:18-25.

30 Hajjar RR, Atli T, Al-Mandhari Z, et al. Prevalence of aging population in the middle East and its implications on cancer incidence and care. Ann Oncol 2013;24 Suppl 7:vii11-24.

31 Huijer HA-S, Bejjani R, Fares S. Quality of care, spirituality, relationships and finances in older adult palliative care patients in Lebanon. Ann Palliat Med 2019;8:551-8.

32 Chemali Z, Chahine LM, Sibai AM. Older adult care in Lebanon: towards stronger and sustainable reforms. Eastern Mediterranean health journal $=$ La revue de sante de la Mediterranee orientale = alMajallah al-sihhiyah li-sharq al-mutawassit 2008;14:1466-76.

33 Hussein S, Ismail M. Ageing and elderly care in the Arab region: policy challenges and opportunities. Ageing Int 2017;42:274-89.

34 Sibai AM, Yamout R, eds. Family-Based old-age care in Arab countries: between tradition and modernity. Berlin, Heidelberg: Springer, 2012.

35 Zeinah GFA, Al-Kindi SG, Hassan AA. Middle East experience in


2013;30:94-9.

36 Zhang N, Ning X-hong, Zhu M-lei, et al. Attitudes towards advance care planning and healthcare autonomy among community-dwelling older adults in Beijing, China. Biomed Res Int 2015;2015:1-10.

37 Koenig HG, Büssing A. The Duke university religion index (DUREL) a five-item measure for use in epidemological studies. Religions 2010;1:78-85

38 Elbejjani M, Wahab K, El Hachem R, et al. Knowledge and attitude towards Alzheimer 's disease and related dementias in a low- to middle-income country: a cross-sectional survey among Lebanese middle-aged and older adults. Psychogeriatrics 2021;21:699-708.

39 Carpenter BD, Balsis S, Otilingam PG, et al. The Alzheimer's Disease Knowledge Scale: Development and Psychometric Properties. Gerontologist 2009;49:236-47.

40 Khonje V, Milligan C, Yako Y, et al. Knowledge, attitudes and beliefs about dementia in an urban Xhosa-speaking community in South Africa. Advances in Alzheimer's Disease 2015;04:21-36.
41 Dumit NY, Abboud S, Massouh A, et al. Role of the Lebanese family caregivers in cardiac self-care: a collective approach. J Clin Nurs 2015;24:3318-26.

42 de Pentheny O'Kelly C, Urch C, Brown EA. The impact of culture and religion on truth telling at the end of life. Nephrology Dialysis Transplantation 2011;26:3838-42.10.1093/ndt/gfr630

43 Ayoub F, Fares Y, Fares J. The psychological attitude of patients toward health practitioners in Lebanon. $N$ Am J Med Sci 2015;7:452.

44 Mouhawej MC, Maalouf-Haddad N, Tohmé A, et al. Cultural challenges in implementing palliative services in Lebanon. Palliat Med Hosp Care Open J 2017;SE:S15-18.

45 Rida M-AT, Zeineldine S. Palliative care in Lebanon: the law and the patient. J Med Liban 2013;61:237-9.

46 Edinger W, Smucker DR. Outpatients' attitudes regarding advance directives. J Fam Pract 1992;35:650-4.

47 Carr D, Moorman SM, eds. End-of-life treatment preferences among older adults: an assessment of psychosocial influences 1. sociological forum. Hoboken: Wiley Online Library, 2009.

48 McPherson CJ, Wilson KG, Murray MA. Feeling like a burden to others: a systematic review focusing on the end of life. Palliat Med 2007;21:115-28.

49 Levi BH, Dellasega C, Whitehead M, et al. What influences individuals to engage in advance care planning? Am J Hosp Palliat Care 2010;27:306-12.

50 Romain M, Sprung CL. Approaches to patients and families with strong religious beliefs regarding end-of-life care. Curr Opin Crit Care 2014;20:668-72.

51 Bülow $\mathrm{H}-\mathrm{H}$, Sprung $\mathrm{CL}$, Baras M, et al. Are religion and religiosity important to end-of-life decisions and patient autonomy in the ICU? the Ethicatt study. Intensive Care Med 2012;38:1126-33.

52 Pereira-Salgado A, Mader P, O'Callaghan C, et al. Religious leaders' perceptions of advance care planning: a secondary analysis of interviews with Buddhist, Christian, Hindu, Islamic, Jewish, Sikh and Bahá'í leaders. BMC Palliat Care 2017;16:1-10. 